\title{
Examining the Usability of Touch Screen Gestures for Elderly People
}

\author{
Doris Cáliz ${ }^{1(凶)}$, Xavier Alamán ${ }^{2}$, Loic Martínez ${ }^{1}$, Richart Cáliz ${ }^{3}$, \\ Carlos Terán $^{3}$, and Verónica Peñafiel ${ }^{4}$ \\ ${ }^{1}$ CETTICO Research Group, ETSIINF, Polytechnic University of Madrid, \\ Campus de Montegancedo, 28660 Boadilla del Monte, Madrid, Spain \\ doriscalizramos@outlook.com, loic@f.upm.es \\ 2 Department of Computer Science, Autonomous University of Madrid, \\ C/Francisco Tomás y Valiente, 11, 28049 Madrid, Spain \\ Xavier.Alaman@uam.es \\ ${ }^{3}$ FIS Group, Departamento de Informática y Ciencias de la \\ Computación (DICC). EPN, Escuela Politécnica Nacional, \\ Ladrón de Guevara E11-25 y, Andalucía, Quito, Ecuador \\ richartharold@hotmail.com, carlos.teran@cobiscorp.com \\ 4 Datos Análisis Estadístico. Análisis de datos, \\ Allcuquiro 2-75 y, Paseo de los Cañaris, Cuenca, Ecuador \\ veropenafelm@gmail. com
}

\begin{abstract}
This paper presents an experimental study to assess the capabilities of older adults to interact with multi-touch surfaces. The study involved 100 elderly people between 61-92 years old. We selected two different elderly centres in Madrid, with different characteristics in terms of income level. The "Gesture Games" tool was used because it allows experimenting with the seven more used multi-touch gestures: Tap, Double tap, Long press, Drag, Scale up, Scale down and One-finger rotation. The analysis of the data showed that older adults have total capacity to execute these seven tasks. Some of the tasks, such as "scale down" and "scale up" were found easier for them, while other tasks, such as "double tap" were more difficult.
\end{abstract}

\section{Introduction and Previous Work}

As people age, their cognitive and/or physical abilities start to degrade and could prevent them from properly using a tablet. For this reason is important to study what kind of skills have the elderly people when they use multi-touch superficies such as smartphones, tablets and netbooks [1]. According to Abascal, what older users expect from mobile communications is not very different from what the generic user expects from these services: mostly, fully reliable personal communications and services to improve, as much as possible, safety and quality of life [2].

There are already several works studying these problems. For example, "Anshin", is a software application for mobile devices to prevent solitary death and to provide a 
social network. The tool use touch gestures (select and drag) to change the configuration [3]. "Dance! Don't Fall" is an application for elderly people that teach them to dance, in which drag gestures are intensively used. The project senSAVEr is a system that provides a continuous mobile health monitoring service for people with hypertension. The interface was characterized by displays of graphical symbols and animations which contain the possibility to check parameter values [4] using touch gestures. Moderne Kijkbuis' is an entertainment application for elderly with physical and/or cognitive impairments in which the elderly had major difficulties pressing two dimensional buttons with basic touch gestures [5].

This paper presents an experimental study to assess the capabilities of older adults to interact with multi-touch surfaces.

\section{Experimental Study}

The overall objective of this experimental study was to identify the skills of elderly adults to handle tablets with suitable gestures, taking into account their limitations, in order to determine the best suited to applications for this population segment [6]. Using the Metric Question (GQM) Meta-template, our goal can be defined in the following way: to analyse a set of multi-touch gestures for the purpose of assessing their suitability from the point of view of the usability of multi-touch technologies in the context of the elderly people (Fig. 1).

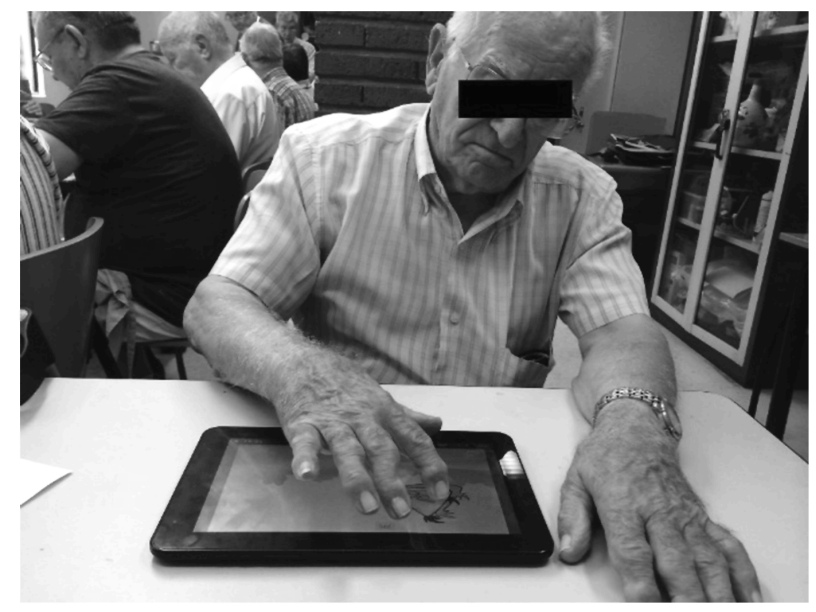

Fig. 1. Elderly people during the workshop.

Gender and the group age were the two main variables considered independent. Execution time and success rate were the two dependent variables for each task (Tap, Double tap, Long press, Drag, Scale up, Scale down, One-finger rotation). Consequently, the hypotheses to be tested statistically, as defined for each task performed (type of gesture), were formulated as follows: H1, completion time of task $\mathrm{k}$ is not 
affected by gender; $\mathrm{H} 2$, completion time of task $\mathrm{k}$ is not affected by age group; $\mathrm{H} 3$, the degree of success for task $\mathrm{k}$ is independent of gender; $\mathrm{H} 4$, the degree of success for task $\mathrm{k}$ is independent of age group; H5, the degree of success is independent of the task.

In order to test these hypotheses, we measured the manipulation time of each gesture as well as it success, with the ultimate goal of obtaining a set of guidelines specifically focused on designing touch-enabled applications for elderly people.

\subsection{Participants}

The evaluation was performed with a total of 100 seniors in two different centres of the Community of Madrid, Spain. The Sagasta Centre is located in an area with a high purchasing power while the San Blas Centre is located in a low-income area. In each centre 50 seniors participated, 25 women and 25 men, making a total of 100 participants. The age varied between 61 and 92 years.

\subsection{Equipment}

The interaction framework for the experiment was implemented in Java using JMonkeyEngine SDK v.3.Obeta. The application name is Gestures Games [7]. The device used for the experiment was a Szenio 10.1, 1 GB RAM, 32 GB tablet with Android 4.2.2 with capacitive multi-touch screens.

\subsection{Procedure}

For each task, the elderly were given a 5-minute learning phase with an instructor. The task were performed in a sequential order by each participant. All the participants hold the tablet in the same way. The same instructor carried out all the experiments. The experimental platform then asked them to perform the task without any assistance. They had to perform three repetitions of each gesture under specific conditions. When the gesture was completed successfully, the platform gave a positive audio-visual feedback. If the instructor saw that the participant did not carry out the task in a given time, it was marked as undone and the elderly went on to the next one. For each interaction, the system recorded the start time (seconds needed to go into action after the visual stimulus was shown), completion time, success (performed correctly or incorrectly), and the number of contacts with the surface (in order to detect unsuccessful actions or whether the user had made any attempt to interact). A qualitative analysis was also carried out from the notes taken by an external observer during the experimental sessions.

\subsection{Tasks}

There were 7 tasks that the elderly had to perform: 
Task 1: Tap. A static image of an animal appears in a random position on the screen (see Fig. 2). Participants are requested to tap on the target image in order to pass the test.

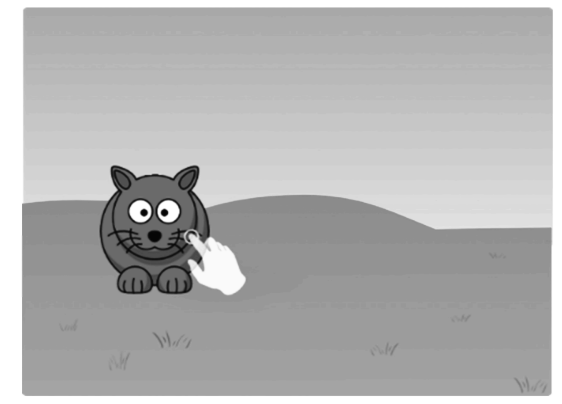

Fig. 2. Example of a simple tap, double tap or long pressed test

Task 2: Double Tap. A static image of an animal appears in a random position on the screen (see Fig. 2). Participants are requested to double tap on the target image with one finger.

Task 3: Long Press. A static image of an animal appears in a random position on the screen (see Fig. 2). Participants are requested to carry out a long press gesture on the target image until the target disappears.

Task 4: Drag. A static image of an animal appears in a random position on the screen and the same (reference) image appears in a white profile in another random position, always at the same distance (Fig. 3). Participants are requested to drag the target to the reference image with one finger.

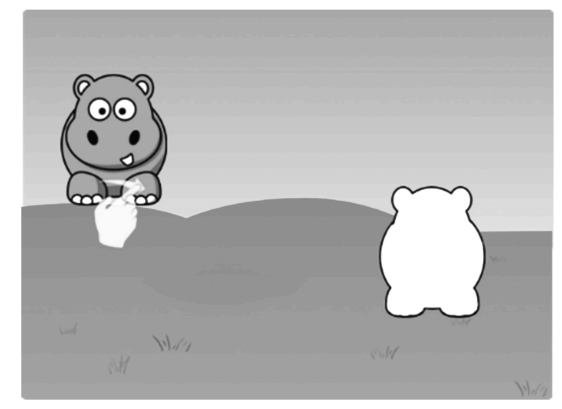

Fig. 3. Example of a drag test.

Task 5: Scale up. A static image of an animal appears in the centre of the screen within a similar but 1.5 times larger reference shape. Participants are requested to scale up the target image to the size of the reference shape. 
Task 6: Scale down. A static image of an animal appears in the centre of the screen superimposed on a similar reference shape half its size (see Fig. 4-b). Participants are requested to scale down the target image.

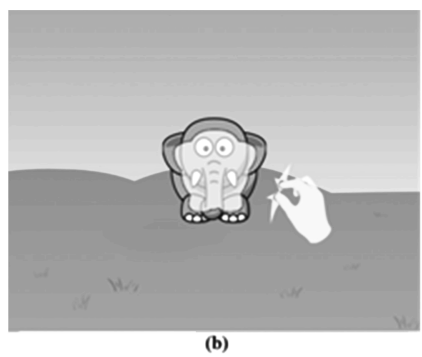

(b)

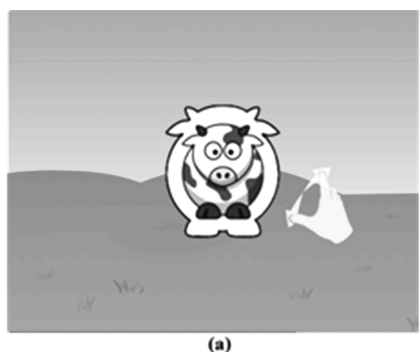

(a)

Fig. 4. Example of a scale test: (a) scale up and (b) scale down.

Task 7: One-finger rotation. A static image of an animal appears in the centre of the screen in front of a blank profile of the same image in a different orientation. Participants are requested to rotate the target image to the position of the reference image.

\section{Results}

We have selected the following aspects to analyse: number of tasks completed, rate of task compliance (All, Sex, Age, Centre, Experience), ratio of task compliance, task completing time (All, Sex, age, Centre, Experience), and ratio of task completing times. Each participant has tried at least two of the gestures proposed in the application: $48.51 \%$ of participants have achieved successful completion of at least two repetitions of the same task.

\section{- Task success}

The incidence of success in fulfilling tasks using different gestures according to sex and age showed no statistically significant associations. However, the gesture "one-finger rotation", in the case of analysis by centres, presented an association, as exposed by Chi-square variable test $(\mathrm{p}<0.05)$. Previous experience in the use of smartphones marked differences in the incidence of performance in the tasks "tap", "double tap", "long press" and "drag" ( $\mathrm{p}<0.05)$. (See Table 1).

\section{- Inter-gesture correlations in task success}

They were found moderate-low correlations between the success in the "tap" task and success in the "double tap" and "one-finger rotation".

The correct accomplishment of the "double tap" has a strong correlation with the "long press" task. Furthermore, the "long press" task has a moderate-low correlation with "drag" and with "scale-up" tasks. Success in the "Drag" task is positively correlated with "scale-up", "scale-down" and "one-finger rotation". In addition, "scale-up" and "scale-down" tasks are correlated with each other. 
Table 1. Test x2, incidence of success with gesture VS Gender, Centre, Age, Previous Experience

\begin{tabular}{l|l|l|l|l|l|l|l|l}
\hline \multirow{2}{*}{ Gesture } & \multicolumn{3}{l}{ Gender } & \multicolumn{2}{l|}{ Centre } & \multicolumn{2}{l}{ Age } & \multicolumn{2}{l}{ Experience } \\
\cline { 2 - 10 } & $\mathrm{x} 2$ & $\mathrm{p}$ & $\mathrm{x} 2$ & $\mathrm{p}$ & $\mathrm{x} 2$ & $\mathrm{p}$ & $\mathrm{x} 2$ & $\mathrm{p}$ \\
\hline Tap & 0,000 & 0,992 & 1,282 & 0,257 & 2,248 & 0,134 & $8,189^{*}$ & 0,004 \\
\hline Double tap & 0,209 & 0,648 & 0,246 & 0,620 & 3,749 & 0,053 & $6,831^{*}$ & 0,009 \\
\hline Long press & 0,323 & 0,570 & 0,469 & 0,494 & 0,009 & 0,926 & $5,755^{*}$ & 0,016 \\
\hline Drag & 0,027 & 0,869 & 0,190 & 0,663 & 0,153 & 0,696 & $6,815^{*}$ & 0,009 \\
\hline Scale-Up & 1,575 & 0,209 & 2,000 & 0,157 & 2,165 & 0,141 & 2,643 & 0,104 \\
\hline Scale-Down & 0,034 & 0,853 & 0,000 & 0,989 & 0,002 & 0,966 & 0,034 & 0,853 \\
\hline One-finger rot. & 0,008 & 0,927 & 5,983 & $0,036^{*}$ & 0,050 & 0,823 & 0,001 & 0,978 \\
\hline
\end{tabular}

Note: * significative correlation $(p<0.05)$

Finally, "scale-up" and "scale-down" are strongly correlated with the success in the "one-finger rotation" task (see Table 2).

Table 2. Inter-gesture correlations

\begin{tabular}{|c|c|c|c|c|c|c|c|}
\hline & & Double-tap & Long press & Drag & Scale up & Scale down & $\begin{array}{l}\text { One-finger } \\
\text { rotation }\end{array}$ \\
\hline \multirow[t]{2}{*}{ Tap } & v & 0,391 & 0,187 & 0,043 & 0,092 & 0,077 & 0,243 \\
\hline & $\mathrm{p}$ & $0,00^{* *}$ & 0,060 & 0,203 & 0,354 & 0,438 & $0,015^{*}$ \\
\hline \multirow[t]{2}{*}{ Double tap } & v & & 0,333 & 0,018 & 0,097 & 0,097 & 0,084 \\
\hline & $\mathrm{p}$ & & $0,010^{*}$ & 0,237 & 0,331 & 0,331 & 0,401 \\
\hline \multirow[t]{2}{*}{ Long press } & v & & & 0,333 & 0,277 & 0,102 & 0,076 \\
\hline & $\mathrm{p}$ & & & $0,001 * *$ & $0,050^{*}$ & 0,304 & 0,445 \\
\hline \multirow[t]{2}{*}{ Drag } & V & & & & 0,295 & 0,49 & 0,249 \\
\hline & $\mathrm{p}$ & & & & $0,003^{*}$ & $0,000^{* * *}$ & $0,013^{*}$ \\
\hline \multirow[t]{2}{*}{ Scale-up } & v & & & & & 0,295 & 0,653 \\
\hline & $\mathrm{p}$ & & & & & $0,003^{*}$ & $0,045^{*}$ \\
\hline \multirow[t]{2}{*}{ Scale-down } & v & & & & & & 0,653 \\
\hline & $\mathrm{p}$ & & & & & & $0,045^{*}$ \\
\hline
\end{tabular}

Note: $* \mathrm{p}<0.05$ (moderate corr.) ${ }^{* *} \mathrm{p}<0.001$ (strong corr.)

\section{- Task completing time}

The tasks with a longer average completion time corresponds to the gesture of "one-finger rotation" with a value of $5.42 \mathrm{~s}(\mathrm{SD}=4.16 \mathrm{~s})$, followed by the activity of "drag" with an average value of $4,96 \mathrm{~s}(\mathrm{SD}=2.88)$. The activity with a shorter completion time was the activity of "two taps", although this action had also the lower incidence of success.

The activities that showed highest variances were "tap" and "scale-down" (see Table 3). 
Table 3. Completing time of tasks

\begin{tabular}{l|l|l|l|l|l}
\hline Gesture & Min. & Max. & Average & $\begin{array}{l}\text { Standard } \\
\text { deviation }\end{array}$ & Variance \\
\hline Tap & 0,72 & 78,54 & 4,56 & 9,19 & 84,40 \\
\hline Double tap & 0,72 & 6,83 & 2,14 & 1,22 & 1,49 \\
\hline Long press & 1,35 & 9,71 & 3,23 & 1,78 & 3,17 \\
\hline Drag & 1,54 & 16,54 & 4,96 & 2,88 & 8,30 \\
\hline Scale up & 0,68 & 22,43 & 2,71 & 2,72 & 7,37 \\
\hline Scale down & 0,92 & 50,87 & 4,05 & 5,47 & 29,91 \\
\hline One-finger rotation & 1,32 & 29,83 & 5,42 & 4,16 & 17,34 \\
\hline
\end{tabular}

\section{- Task completing time correlations with Gender, Age Centre and previous touch screen experience.}

The time of completion of the tasks showed significant differences between men and women in the gestures "tap", "long press" and "drag" $(\mathrm{p}<0.05)$. In terms of age, significant differences were found between the group of people over 71 years and the group of people under 71 years of age in the gesture "tap". The previous experience with the use of smartphones showed a significant difference in the gesture of "scale down" (see Table 4).

Table 4. Mann-Whitney $U$ test on task completing time

\begin{tabular}{l|l|l|l|l}
\hline \multirow{2}{*}{ Gesture } & Gender & Age & Centre & Experience \\
\cline { 2 - 5 } & $\mathrm{P}$ & $\mathrm{p}$ & $\mathrm{p}$ & $\mathrm{P}$ \\
\hline Tap & $0,039^{*}$ & $0,050^{*}$ & 0,932 & 0,333 \\
\hline Double tap & 0,274 & 0,405 & 0,674 & 0,378 \\
\hline Long press & $0,004^{*}$ & 0,456 & 0,992 & 0,493 \\
\hline Drag & $0,022^{*}$ & 0,218 & 0,660 & 0,483 \\
\hline Scale up & 0,924 & 0,249 & 0,585 & 0,399 \\
\hline Scale down & 0,338 & 0,059 & 0,263 & $0,042^{*}$ \\
\hline One-finger rotation & 0,307 & 0,136 & 0,422 & 0,383 \\
\hline Note: * significative correlation, $p<0.05$
\end{tabular}

The women showed an average completion time greater than that of men in the gestures related to "long press" and "drag", with averages of $3894.5 \mathrm{~ms}$ (SD = $2224.2 \mathrm{~ms}$ ), and $5299.9 \mathrm{~ms}(\mathrm{DE}=2589.9 \mathrm{~ms}$ ) respectively, compared to $2694.5 \mathrm{~ms}$ ( $\mathrm{DE}=1067.7 \mathrm{~ms}$ ) showed by men for the "long press" and 4688.0 ( $\mathrm{SD}=3084,5 \mathrm{~ms}$ ) for "drag". On the other hand the time required to complete the activity "tap" was greater for men with an average of $5221.7 \mathrm{~ms}(\mathrm{SD}=12174.4 \mathrm{~ms})$ (See Fig. 5).

The group of people under 71 years showed smaller completion times with an average difference of $3426,23 \mathrm{~ms}$. The behaviour for each task is shown in Fig. 6.

The behaviour of the time taken for the completion of tasks, depending on the centre or depending on the previous experience of the elderly, are shown in Figs. 7 and 8. 


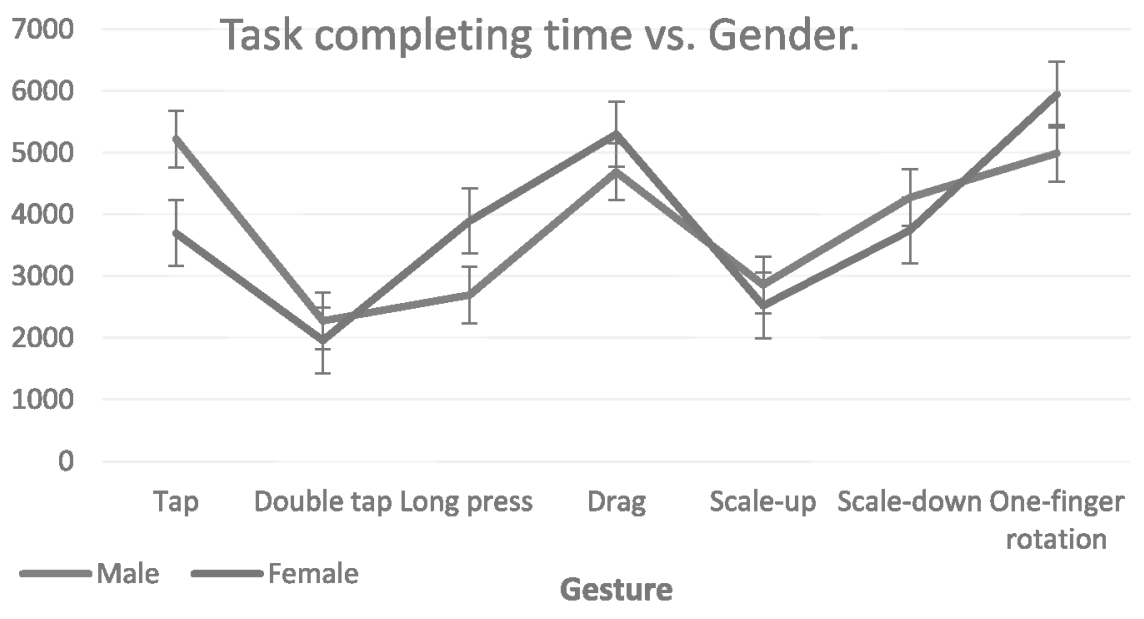

Fig. 5. Task completing time vs. gender.

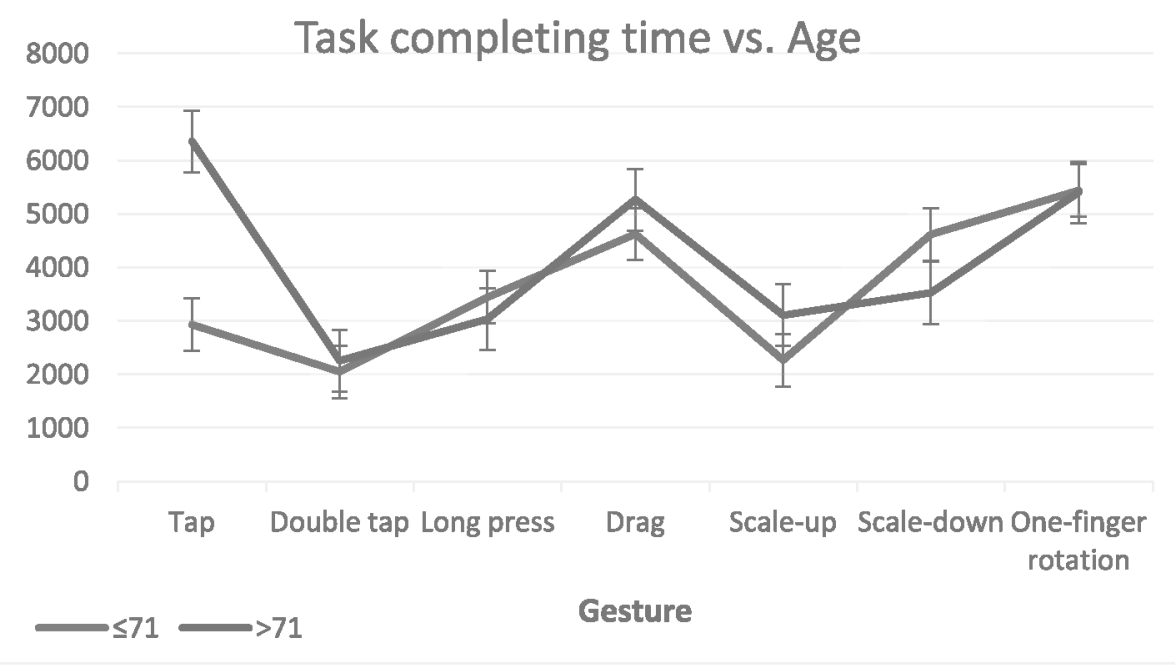

Fig. 6. Task completing time vs. age

The action "scale down" correlated with the previous experience of the elderly: people with previous experience in management smartphones used an average of $4760.2 \mathrm{~ms}$ $(\mathrm{DE}=6940,2 \mathrm{~s})$ compared to $3140.1 \mathrm{~ms}(\mathrm{DE}=2427,9 \mathrm{~s})$ for the inexperienced people. 


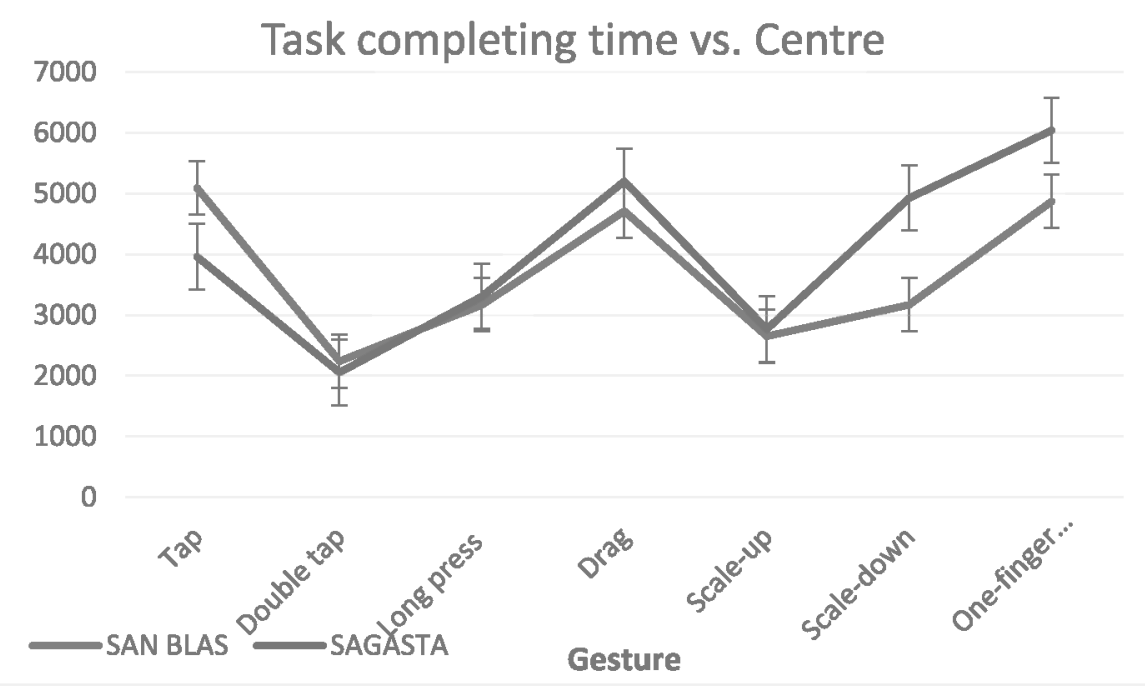

Fig. 7. Task completing time vs. centre

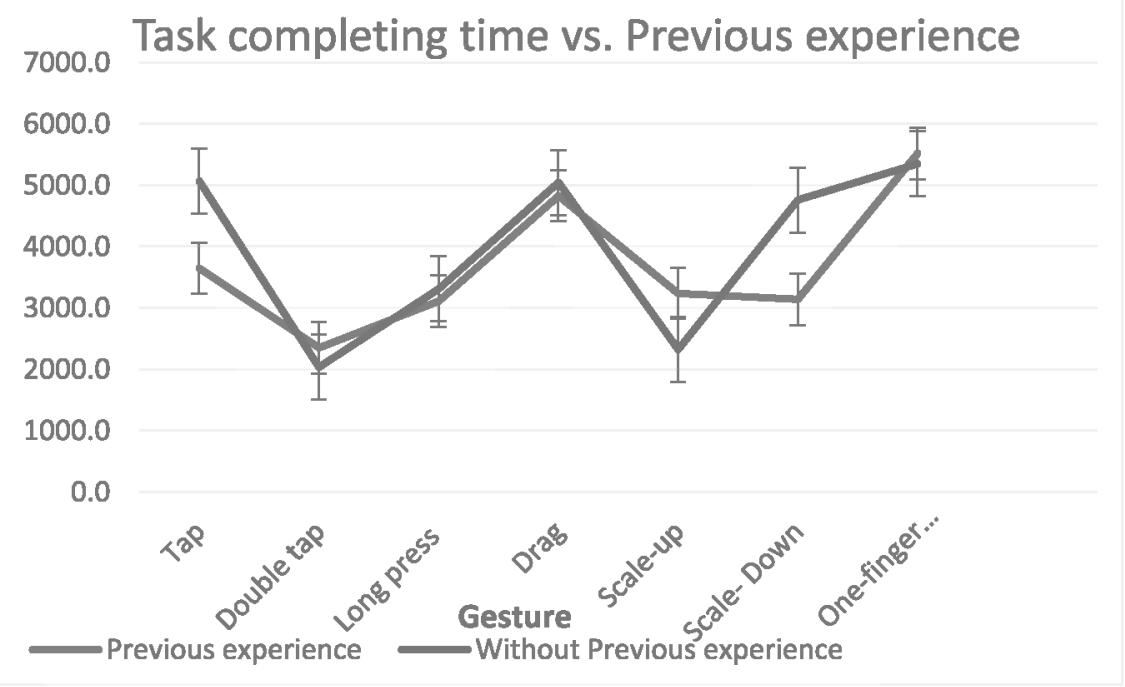

Fig. 8. Task completing time vs. previous experience

\section{- Inter-gesture correlation in task completing times}

The time taken to complete the task "tap" is slightly correlated with the time spent performing "double tap" and "drag". The time taken to complete the task "double tap" has a moderate-low correlation with the "long press" and "scale up". In addition "Long 
press" is positively correlated with "drag". The time spent in the action "drag" has a moderate-low correlation with "scale up", "scale down" and "one-finger rotation". Scale up and "scale down" are moderately correlated (see Table 5).

Table 5. Inter-gesture correlation in task completing times

\begin{tabular}{l|l|l|l|l|l|l|l}
\hline \multicolumn{2}{l|}{} & Double-tap & Long press & Drag & Scale up & Scale down & $\begin{array}{l}\text { One-finger } \\
\text { rotation }\end{array}$ \\
\hline \multirow{2}{*}{ Tap } & $\mathrm{Rh}$ & $0,270^{*}$ & 0,208 & $0,246^{*}$ & 0,159 & 0,131 & $-0,057$ \\
\cline { 2 - 8 } & $\mathrm{p}$ & 0,032 & 0,094 & 0,031 & 0,165 & 0,255 & 0,632 \\
\hline \multirow{2}{*}{ Double tap } & $\mathrm{Rh}$ & & $0,335^{* *}$ & 0,208 & $0,339^{* *}$ & 0,189 & $-0,012$ \\
\cline { 2 - 8 } & $\mathrm{p}$ & & 0,007 & 0,085 & 0,004 & 0,120 & 0,927 \\
\hline \multirow{2}{*}{ Long press } & $\mathrm{Rh}$ & & & $0,333^{* *}$ & 0,165 & 0,157 & $-0,004$ \\
\cline { 2 - 8 } & $\mathrm{p}$ & & & 0,003 & 0,143 & 0,168 & 0,976 \\
\hline \multirow{2}{*}{ Drag } & $\mathrm{Rh}$ & & & & $0,424^{* *}$ & $0,254^{*}$ & $0,349^{* *}$ \\
\cline { 2 - 8 } & $\mathrm{p}$ & & & & 0,000 & 0,013 & 0,001 \\
\hline \multirow{2}{*}{ Scale-up } & $\mathrm{Rh}$ & & & & & $0,342^{* *}$ & 0,176 \\
\cline { 2 - 8 } & $\mathrm{p}$ & & & & & 0,001 & 0,100 \\
\hline
\end{tabular}

Note: correlation $* \mathrm{p}<0,05 * * \mathrm{p}<0,001$

\section{Conclusions, Results and Future Work}

After this work we are able to answer the fundamental question "Are elderly people ready for multi-touch technology?" The answer is definitely affirmative, provided certain issues are dealt with. About the question "What multi-touch gestures are the people between 61 and 92 years of age able to use?" we found that they are capable of performing all gestures. We showed that not all the tasks are equally feasible but elderly people are able to execute with different levels of complexity. Current applications for elderly people might be missing the opportunity to provide richer gestures within elderly people abilities, and could be using a gesture that is notoriously difficult for them (e.g., double tap, tap, long pressed). The special elderly people skills that we found in this test, was that elderly people can perform easily scale down ( $99 \%$ success) and scale up (98\% success) gestures. Consequently, interaction designers have an opportunity to broaden the scope of their interfaces when creating future applications using these especial abilities. The quantitative results also show that there are still challenging gestures for elderly people (double tap, tap, long pressed) with relative's low success rates ranging. These gestures have to be discussed in the context of the interaction aids or design guidelines that application designers should take into account if these touch interactions are included in future applications. Designers would be improve the way to execute these gestures in to do easily to use or execute these tasks for this special segment of population. For example, increasing the time between the first tap and the second (for the double tap gesture), so that they have time to react and this fit their actual motor abilities. 
In addition to the automatic data logging that was performed to measure completion times and degree of success, an external observer gathered valuable information regarding the behaviour of elderly people during the experiments. These observations revealed different problems that will now be described.

The task was more difficult for them was the double tap, as it is well known that motor skills at their age can be somewhat severed, and possibly are not flexible enough to perform two taps in a short time interval. The task they preferred, according to the data collected and the final questionnaire, was to scale up and scale down, possibly because in this task they can use both hands on the tablet and this gives them security and firmness.

Based on this research, future work have be done in the following areas:

- Incorporating smart elements on mobile applications what can react with tutorials or modify the appearance of the application. This would allow the application to be used by different types of users, including the elderly.

- Expanding the scope of the study to other domains or types of applications and for other types of devices.

- Applying artificial intelligence elements where the tool is able to understand and learn the user behaviour in order to formulate the criteria for improvement.

- Incorporation of audio-visual tools into the assessment tool to better enable user interaction.

\section{References}

1. Cáliz, D., Alamán, X.: Usability evaluation method for mobile applications for the elderly: a methodological proposal. In: Pecchia, L., Chen, L.L., Nugent, C., Bravo, J. (eds.) IWAAL 2014. LNCS, vol. 8868, pp. 252-260. Springer, Heidelberg (2014)

2. Díaz-Bossini, J., Moreno, L.: Accessibility to mobile interfaces for older people. Procedia Comput. Sci. 27, 57-66 (2013)

3. Takami, S., Torii, I., Ishii, N.: Development of system for prevention of solitary death with mobile devices. Procedia Comput. Sci. 35, 1193-1201 (2014)

4. Lorenz, A., Oppermann, R.: Mobile health monitoring for the elderly: Designing for diversity. Pervasive Mob. Comput. 5(5), 478-495 (2009)

5. Muskens, L., van Lent, R., Vijfvinkel, A., van Cann, P., Shahid, S.: Never too old to use a tablet: designing tablet applications for the cognitively and physically impaired elderly. In: Miesenberger, K., Fels, D., Archambault, D., Peňáz, P., Zagler, W. (eds.) ICCHP 2014, Part I. LNCS, vol. 8547, pp. 391-398. Springer, Heidelberg (2014)

6. Nacher, V., Jaen, J., Navarro, E., Catala, A., González, P.: Multi-touch gestures for pre-kindergarten children. J. Hum. Comput. Stud. 73, 37-51 (2015)

7. Guía, E.D., Lozano, M.D., Penichet, V.M.R.: Co-StiCap: Sistema Basado en Interfaces de Usuario Distribuidas y Tangibles Para Mejorar las Capacidades Cognitivas en Niños con TDAH. Interacción, enseñanza y Aprendiz, 61-68 (2014) 